\title{
ERS guidelines, statements and technical standards published in the ERJ in 2014: a year in review
}

\author{
Guy G. Brusselle $e^{1,2}$ and Mina Gaga ${ }^{3}$
}

Affiliations: 'Dept of Respiratory Medicine, Ghent University Hospital, Ghent, Belgium. ${ }^{2}$ Dept of Epidemiology and Respiratory Medicine, Erasmus Medical Center Rotterdam, Rotterdam, The Netherlands. ${ }^{3} 7$ th Respiratory Medicine Dept and Asthma Centre, Athens Chest Hospital "Sotiria”, Athens, Greece.

Correspondence: Guy G. Brusselle, Dept of Respiratory Medicine, Ghent University Hospital, De Pintelaan 185, B-9000 Ghent, Belgium. E-mail: guy.brusselledugent.be

@ERSpublications

2014: a year in review of ERS guidelines, statements and technical standards http://ow.ly/HnGDs

The number of scientific journals and the number of publications regarding biomedical research is rapidly increasing at an almost exponential rate. Therefore, there is a huge need for physicians, allied healthcare professionals, researchers and patients to have access to clear and valid syntheses of the available evidence on the management of specific diseases or research topics within respiratory medicine and science. There are at least two different approaches to provide a synopsis on the diagnosis, prevention or treatment of a respiratory disease or health problem. In the first approach, one author or a few authors provide a narrative review of the management of the respiratory disease of interest, without performing a comprehensive systematic review of the literature and without providing explicit recommendations for clinical practice. Although the authors are often experts in their field, they do not aim to search, grasp and cite all available evidence, implicating that this approach is prone to systematic errors or biases (encompassing publication bias and reporting bias). In the second approach, a multidisciplinary panel of experts (e.g. a European Respiratory Society (ERS) Task Force) searches in a systematic and transparent manner for all available evidence on the respiratory disease, the diagnostic test or the treatment of interest. Within ERS Task Forces, the Chairs and members are assisted in the methodological processes required for the development of statements and guidelines by experts (i.e. methodologists). Whereas the comprehensive scientific review of the literature by an ERS Task Force forms the basis of an official ERS statement or technical standard, the development of an ERS clinical practice guideline requires several crucial additional steps. The aims of this editorial are three-fold: 1) to explain the differences between the three possible outcomes of an ERS Task Force; a guideline, a statement or a technical standard; 2) to provide a concise overview of several important ERS Task Force documents published in the European Respiratory Journal (ERJ) in the past year [1-6]; and 3) to give some insights into the development and publication processes of ERS guidelines, statements and technical standards.

The aim of an ERS Task Force is to develop a high-quality trustworthy document on the management (diagnosis, prevention and/or treatment) of a respiratory disease in a timely manner (i.e. within 2 years). The final document can either be a clinical practice guideline, a statement or a technical standard. The difference between a guideline or statement, on the one hand, and a technical standard, on the other hand, mainly depends on the research question addressed by the Task Force. Technical standards address questions regarding "how to" perform a test or procedure. In contrast, clinical practice guidelines give recommendations about "in whom" and "under what circumstances" a test or procedure should be applied. As an example, the ERS and American Thoracic Society (ATS) have jointly published a technical

Received: Dec 312014 | Accepted: Jan 062015

G.G. Brusselle is the ERS Guidelines Director and M. Gaga is an Associate Editor for the European Respiratory Journal.

Conflict of interest: Disclosures can be found alongside the online version of this article at erj.ersjournals.com

Copyright @ERS 2015 
standard on how to measure exhaled nitric oxide [7]. However, the recommendations on the use and interpretation of exhaled nitric oxide levels for clinical applications have been specified in a separate clinical practice guideline [8]. The difference between a guideline and a statement is based upon the methodological requirements: whereas a comprehensive scientific review of the literature by an ERS Task Force forms the basis of an official ERS abatement, the development of an ERS clinical practice guideline requires, in addition to systematic reviews, grading of the quality of the evidence and grading of the strength of the recommendations $[9,10]$. The ERS has chosen to apply the GRADE (Grading of Recommendations, Assessment, Development and Evaluation) methodology to develop guidelines, since the GRADE system has many merits. GRADE makes a clear separation between the quality of evidence and the strength of the recommendations, and the process of moving from evidence to recommendations is transparent (and summarised in evidence tables, which are published together with the clinical practice guideline as supplementary material).

In 2014, several important official ERS Task Force reports were published in the ERJ [1-6]. These official ERS documents have been developed either by an single ERS Task Force or by a joint Task Force, whereby the ERS collaborates intensively with a sister society such as the ATS. In the December 2014 issue of the ERJ, Holland et al. [1] published the ERS/ATS technical standard on field walking tests in chronic respiratory disease. Field walking tests, such as the 6-min walk test (6MWT), are important tools to evaluate exercise capacity and assess prognosis in adult patients with chronic respiratory diseases such as chronic obstructive pulmonary disease and pulmonary arterial hypertension. However, since the 6-min walking distance is very sensitive to variations in methodology (e.g. use of encouragement, provision of supplemental oxygen, use of wheeled walkers or changes in track length and layout), the ERS/ATS technical standard provides much needed standard operating procedures on how to perform the 6MWT [1]. Importantly, since there is strong evidence of a learning effect for the 6MWT, the ERS/ATS technical standard proposes that two tests should be performed at baseline when the 6MWT is used to measure change over time (e.g. to evaluate treatment response). In an accompanying systematic review on field walking tests, the ERS/ATS Task Force also provided an excellent overview of the measurement properties and interpretation of the 6MWT and other field walking tests, such as the incremental shuttle walk test and the endurance shuttle walk test [2].

In February 2014, CHUnG et al. [3] published the joint international ERS/ATS clinical practice guideline on severe asthma in the ERJ. An asset of this ERS/ATS guideline is the clear and novel definition of severe asthma as well as uncontrolled asthma. Secondly, the ERS/ATS guideline provides practical guidance to physicians on how to evaluate patients with severe asthma, taking into account the clinical heterogeneity of the asthma syndrome and the multiple severe asthma phenotypes. The ERS/ATS guideline on severe asthma provides specific recommendations on treatment with anti-IgE antibody, methotrexate, macrolide antibiotics, antifungal agents and bronchial thermoplasty [3]. Importantly, the ERS/ATS guideline clearly illustrates the need for independent and transparent systematic reviews of the literature for each separate PICO question (Patients, Intervention, Control intervention and Outcomes). As an example we briefly discuss here the PICO question regarding the benefit risk-ratio of bronchial thermoplasty in patients with severe asthma. An advanced PubMed search using the keywords "asthma" and "bronchial thermoplasty (BT)" provided 56 results, giving the false impression that this novel invasive procedure has been investigated in depth in asthma. However, a comprehensive systematic review of the literature by the ERS/ ATS Task Force identified only three randomised trials, of which only one trial specifically enrolled patients with severe asthma (15 patients in each arm of the randomised controlled trial) [11-13]. Moreover, there appeared to be a reporting bias in the original papers describing the effects of bronchial thermoplasty on exacerbations of asthma, since the primary manuscripts focussed on the period starting 6 weeks after the last bronchoscopic procedure, minimising the (serious) adverse events that occurred during the actual treatment period of bronchial thermoplasty. In the supplementary material of the ERS/ ATS guidelines on severe asthma, the significantly increased rate of severe respiratory adverse effects during the bronchoscopic treatment phase (relative risk 8.98 of bronchial thermoplasty compared with sham control or usual care) is clearly described [3, 14]. Taking both the desired and undesired effects of bronchial thermoplasty into account, the ERS/ATS Task Force has correctly made the strong recommendation that, in adults with severe asthma, bronchial thermoplasty should be performed only in the context of an Institutional Review Board-approved registry or clinical study.

In patients with severe asthma with a late onset (i.e. onset of asthma in adulthood), occupational asthma should be part of the differential diagnosis. In 2014, a ERS Task Force report on specific inhalation challenge in the diagnosis of occupational asthma was published by VANDENPLAS et al. [4]. This excellent ERS document provides practical guidance for specific inhalation challenges in the diagnosis of occupational asthma, and is based upon a systematic literature search, a census of active European centres, a Delphi conference and expert consensus. This article details each step of a specific inhalation challenge, 
including safety requirements, techniques for delivering agents, and methods for assessing and interpreting bronchial responses. The limitations of the procedure are also discussed. Testing should only be carried out in hospitals where physicians and healthcare professionals have appropriate expertise. Tests should always include a control challenge, a gradual increase of exposure to the suspected agent, and close monitoring of the patient during the challenge and for at least $6 \mathrm{~h}$ afterwards. In expert centres, excessive reactions provoked by a specific inhalation challenge are rare. A positive response is defined by a fall in forced expiratory volume in $1 \mathrm{~s}\left(\mathrm{FEV}_{1}\right) \geqslant 15 \%$ from baseline. Equivocal reactions can sometimes be clarified by finding changes in nonspecific bronchial responsiveness, sputum eosinophilia or exhaled nitric oxide [4]

In December 2014, panel members of the International Society for Heart and Lung Transplantation (ISHLT)/ATS/ERS bronchiolitis obliterans syndrome (BOS) Task Force published an official clinical practice guideline on the diagnosis and management of BOS [5]. BOS is a major complication of lung transplantation, which is defined as a delayed allograft dysfunction with persistent decline in FEV 1 that is not caused by other known and potentially reversible causes of post-transplant loss of lung function, such as infection, vascular obstruction or mechanical abnormalities. The ISHLT/ATS/ERS BOS guidelines identify several risk factors associated with BOS, provide an algorithm for clinical evaluation of suspected BOS, and formulate specific recommendations about the use of systemic corticosteroids, cyclosporine, tacrolimus, azithromycin and re-transplantation in patients with BOS [5]. Finally, the authors list key unanswered questions and research needs in the prevention and management of BOS.

Last but not least, the ERS Task Force on the European initiative for quality management in lung cancer care provided the first comprehensive study of lung cancer care in Europe [6]. Lung cancer is the most common cause of cancer-related death worldwide and poses a significant respiratory disease burden. The overall aim of the ERS Task Force was to investigate current practice in lung cancer care across Europe. The Task Force undertook four projects: 1) a narrative literature search on quality management of lung cancer; 2) a survey of national and local infrastructure for lung cancer care in Europe; 3) a benchmarking project on the quality of (inter)national lung cancer guidelines in Europe; and 4) a feasibility study of prospective data collection in a pan-European setting. Intriguingly, there appeared to be little peer-reviewed literature on quality management in lung cancer care. The survey revealed important differences in the infrastructure of lung cancer care in Europe. The European guidelines that were assessed displayed wide variation in content and scope, as well as methodological quality. Finally, the feasibility study demonstrated that it is, in principle, feasible to collect prospective demographic and clinical data on patients with lung cancer across Europe, although legal obligations vary among countries [6].

In summary, guidelines, statements and technical standards not only improve clinical decision-making by clinicians and allied healthcare professionals at the individual level, but are also important tools to advance standards of care at the population level. Thus, the target audience of guidelines has expanded from clinicians and patients to health system administrators, funders and regulators. Because of the crucial role of guidelines for clinical practice and organisation of healthcare, the ERS has established a Guidelines Working Group within the ERS Science Council to facilitate the efficient development, dissemination and implementation of excellent, trustworthy guidelines. All ERS members are eligible to apply for funding to set up an ERS Task force. The application process is detailed on the ERS website (www.ersnet.org/ ers-funding/task-forces.html). It helps if ideas are initially discussed within the relevant ERS Assembly in order to delineate the exact aims and scopes of the project, the time frame and the composition of the Task Force. An application form should be completed and sent to the ERS. Since the ERS is actively expanding its methodological capacity, there are unique opportunities for junior ERS members to join specific ERS Task Forces and apply for ERS Fellowships in guideline methodology. Selected ERS candidates will receive appropriate training in conducting systematic reviews, and/or acquire hands-on experience in grading the evidence and formulating the recommendations according to the GRADE approach for the optimal development of guidelines. Importantly, the ERS strongly welcomes collaboration with other (European and non-European) scientific societies to set up joint Task Forces aiming to develop and disseminate novel guidelines. Since multimorbidity is increasing steadily in ageing populations, and since the development of excellent guidelines requires major efforts in people, time and funding, the ERS and other scientific societies should join forces and funds in order to establish multidisciplinary Task Force panels to provide appropriate guidance to physicians and patients on relevant topics in respiratory medicine and science.

\section{References}

1 Holland AE, Spruit MA, Troosters T, et al. An official European Respiratory Society/American Thoracic Society technical standard: field walking tests in chronic respiratory disease. Eur Respir J 2014; 44: 1428-1446.

2 Singh SJ, Puhan MA, Andrianopoulos V, et al. An official systematic review of the European Respiratory Society/ American Thoracic Society: measurement properties of field walking tests in chronic respiratory disease. Eur Respir J 2014; 44: 1447-1478. 
3 Chung KF, Wenzel SE, Brozek JL, et al. International ERS/ATS guidelines on definition, evaluation and treatment of severe asthma. Eur Respir J 2014; 43: 343-373.

4 Vandenplas $\mathrm{O}$, Suojalehto $\mathrm{H}$, Aasen TB, et al. Specific inhalation challenge in the diagnosis of occupational asthma: consensus statement. Eur Respir J 2014; 43: 1573-1587.

5 Meyer KC, Raghu G, Verleden GM, et al. An international ISHLT/ATS/ERS clinical practice guideline: diagnosis and management of bronchiolitis obliterans syndrome. Eur Respir J 2014; 44: 1479-1503.

6 Blum TG, Rich A, Baldwin D, et al. The European initiative for quality management in lung cancer care. Eur Respir J 2014; 43: 1254-1277.

7 American Thoracic Society, European Respiratory Society. ATS/ERS recommendations for standardized procedures for the online and offline measurement of exhaled lower respiratory nitric oxide and nasal nitric oxide, 2005. Am J Respir Crit Care Med 2005; 171: 912-930.

8 Dweik RA, Boggs PB, Erzurum SC, et al. An Official ATS Clinical Practice Guideline: interpretation of exhaled nitric oxide levels (FeNO) for clinical applications. Am J Respir Crit Care Med 2011; 184: 602-615.

9 Brożek JL, Akl EA, Alonso-Coello P, et al. Grading quality of evidence and strength of recommendations in clinical practice guidelines. Allergy 2009; 64: 669-677.

10 Brożek JL, Akl EA, Compalati E, et al. Grading quality of evidence and strength of recommendations in clinical practice guidelines Part 3 of 3. The GRADE approach to developing recommendations. Allergy 2011; 66: 588-595.

11 Cox G, Thomson NC, Rubin AS, et al. Asthma control during the year after bronchial thermoplasty. N Engl J Med 2007; 356: 1327-1237.

12 Castro M, Rubin AS, Laviolette M, et al. Effectiveness and safety of bronchial thermoplasty in the treatment of severe asthma. Am J Respir Crit Care Med 2010; 181: 116-124.

13 Pavord ID, Cox G, Thomson NC, et al. Safety and efficacy of bronchial thermoplasty in symptomatic, severe asthma. Am J Respir Crit Care Med 2007; 176: 1185-1191.

14 Brusselle GG, Kraft M. Trustworthy guidelines on severe asthma thanks to the ERS and ATS. Eur Respir J 2014; 43: 315-318. 